\title{
Hipparcos Luminosities and Asteroseismology
}

\author{
Timothy R. Bedding \\ School of Physics, University of Sydney 2006, Australia
}

\begin{abstract}
Asteroseismology involves using the resonant frequencies of a star to infer details about its internal structure and evolutionary state. Large efforts have been made and continue to be made to measure oscillation frequencies with both ground- and space-based telescopes, with typical precisions of one part in $10^{3}-10^{4}$. However, oscillation frequencies are most useful when accompanied by accurate measurements of the more traditional stellar parameters such as luminosity and effective temperature. The Hipparcos catalogue provides luminosities with precisions of a few percent or better for many oscillating stars. I briefly discuss the importance of Hipparcos measurements for interpreting asteroseismic data on three types of oscillating stars: $\delta$ Scuti variables, rapidly oscillating Ap stars and solar-like stars.
\end{abstract}

\section{Preamble: To 'e' or not to 'e'?}

Because my talk was the last of the day, I chose to begin it with some controversy. I suggested that we heed the urgings of Trimble (1995) to adopt the spelling 'astroseismology.' My basis was a search using the ADS Abstract Service ${ }^{1}$ that showed this spelling in the titles of the first three papers on the subject, by Christensen-Dalsgaard (1984), Gough (1985) and Kurtz (1986). Since all three authors are well-known experts on both stellar oscillations and the English language, I was inclined to accept Trimble's suggestion. I have since checked the original papers and found the ' $e$ ' to be present in all three, so the ADS made a mistake (which they have since corrected). I am happy to retract my suggestion, and refer the interested reader to Gough (1996) for an eloquent and scholarly defence of the ' $e$ '.

Having tracked down the seminal paper on this subject, namely that by Christensen-Dalsgaard (1984), I can do no better than quote his definition of asteroseismology as "the science of using stellar oscillations for the study of the properties of stars, including their internal structure and dynamics." Hipparcos luminosities are important because asteroseismology is most powerful in constraining stellar models when the oscillation frequencies are accompanied by more traditional observables. I will concentrate on three classes of stars for which Hipparcos parallaxes are particularly relevant.

${ }^{1}$ http : //adsabs.harvard.edu/abstract_service.html 


\section{2. $\delta$ Scuti variables}

The $\delta$ Scuti variables are $\mathrm{A}$ and $\mathrm{F}$ stars lying on or near the main sequence, inside the classical instability strip. Recent reviews include those by Guzik (2000) and Handler (2000), as well as the proceedings of a workshop dedicated to these stars (Breger \& Montgomery 2000). Applying asteroseismic techniques to multi-mode $\delta$ Scuti stars has been severely impeded by our inability to identify which oscillation modes are being observed. This difficulty is due both to the mysterious tendency of these stars to oscillate in only a small subset of possible modes, as well as the substantial frequency shifts produced by their fast rotation. The availability of precise Hipparcos parallaxes for some of these stars has not so far generated much progress in interpreting the complicated oscillation spectra (although see Bedding, Kjeldsen, \& Christensen-Dalsgaard 1998 for a discussion of $\kappa^{2}$ Boo).

High-amplitude $\delta$ Scuti stars (HADS) are a subclass for which Hipparcos parallaxes have been extremely valuable. These stars, which constitute about $10 \%$ of the total, pulsate in only one or a few modes (McNamara 1997; Petersen 1998; Alcock et al. 2000). Hipparcos parallaxes were used by Høg \& Petersen (1997) to confirm HADS as otherwise normal stars that follow standard stellar evolution, ruling out alternative low-mass scenarios that had been proposed in the literature. In particular, they showed that the population II field variable SX Phe is well described by standard stellar models and is very similar to the variable blue stragglers in globular clusters.

Petersen \& Høg (1998) used Hipparcos data to construct a period-luminosity relation for HADS, which is in agreement with previous relations derived for clusters. HADS therefore show promise as distance indicators to both the Galactic Bulge (Alcock et al. 2000), as well as to globular clusters and nearby dwarf galaxies (McNamara 2000).

\section{Rapidly oscillating Ap stars}

Lying in the same part of the H-R diagram as the $\delta$ Scuti variables, these are chemically peculiar magnetic stars that oscillate in high-overtone p-modes. Matthews, Kurtz, \& Martinez (1999) have shown that Hipparcos parallaxes for a sample of twelve roAp stars agree reasonably well with parallaxes estimated from asteroseismology. However, there are systematic differences that imply that roAp stars are cooler than indicated by $\mathrm{H} \beta$ photometry, suggesting a need to lower the effective temperature scale of Ap stars. Hopefully, angular diameters from interferometry will soon be able to resolve this issue.

\section{Solar-like oscillations}

The scientific importance of solar-like oscillations can be assessed from the tremendous impact that helioseismology has had on our understanding of the Sun. It seems certain that observations of similar oscillations in other stars will allow new and very strong tests of stellar evolution models. For example, stars only slightly more massive than the Sun are thought to have a convective core for part of their main-sequence lives. The associated mixing will clearly have a 
dramatic influence on the evolution and lifetime of a star, and this will be visible in the oscillation frequencies.

The search for analogues of the 5-minute solar oscillations in other stars finally seems to be bearing fruit. For discussion of a long series of disappointing and tentative results, see reviews by Brown \& Gilliland (1994), Kjeldsen \& Bedding (1995), Heasley et al. (1996) and Bedding \& Kjeldsen (1998). Successes are now starting to appear, with velocity variations in two stars showing excellent evidence for oscillations: Procyon (Martic et al. 1999; Barban et al. 1999) and the G2 subgiant $\beta$ Hyi (Bedding et al. 2000). The subsequent comparisons of observed frequencies with model calculations will rely heavily on Hipparcos parallaxes. This has already been the case for the G0 subgiant $\eta$ Boo, in which good evidence for oscillations was detected by Kjeldsen et al. (1995). At the time, the observed frequencies agreed with the ground-based parallax. Since then, Hipparcos has given a factor of three improvement in parallax precision and maintained the agreement (Bedding et al. 1998), which is an important early success for asteroseismology of solar-like stars.

The recent progress in ground-based observations, as well as results from the star tracker on the otherwise-failed WIRE satellite (Buzasi et al. 2000), illustrate the potential of three upcoming space missions: MOST (Matthews et al. 2000), MONS (Kjeldsen, Bedding, \& Christensen-Dalsgaard 2000) and COROT (Baglin et al. 1998). The prospects for obtaining oscillation measurements on a range of solar-type stars look very good, which therefore makes it vital to obtain as much information about the targets as possible. Hipparcos parallaxes will play a key role, and I strongly urge those planning the GAIA mission not to design out the capability to observe the very brightest stars.

Acknowledgments. For travel support, I thank the Australian Research Council and the Science Foundation for Physics in the University of Sydney.

\section{References}

Alcock, C., et al. 2000, ApJ, 536, 798

Baglin, A., et al. 1998, in IAU Symp. 185, New Eyes to See Inside the Sun and Stars, ed. F.-L. Deubner, J. Christensen-Dalsgaard \& D. W. Kurtz (Dordrecht: Kluwer), 301 (http://www.astrsp-mrs.fr/projets/corot/)

Barban, C., Michel, E., Martic, M., Schmitt, J., Lebrun, J. C., Baglin, A., \& Bertaux, J. L. 1999, A\&A, 350, 617

Bedding, T. R., Butler, R. P., Kjeldsen, H., Baldry, I. K., O'Toole, S. J., Tinney, C. G., Marcy, G. W., Kienzle, F., \& Carrier, F. 2000, submitted to ApJ Letters

Bedding, T. R., \& Kjeldsen, H. 1998, in ASP Conf. Ser. Vol. 154, Tenth Cambridge Workshop on Cool Stars, Stellar Systems and the Sun, ed. R. A. Donahue \& J. A. Bookbinder (San Francisco: ASP), 301

Bedding, T. R., Kjeldsen, H., \& Christensen-Dalsgaard, J., 1998, in ASP Conf. Ser. Vol. 154, Tenth Cambridge Workshop on Cool Stars, Stellar Systems and the Sun, ed. R. A. Donahue \& J. A. Bookbinder (San Francisco: ASP), CD-741 (astro-ph/9709005) 
Breger, M., \& Montgomery, M. 2000, ASP Conf. Ser. Vol. 210, Sixth Vienna Workshop in Astrophysics: Delta Scuti and Related Stars

Brown, T. M., \& Gilliland, R. L. 1994, ARA\&A, 33, 37

Buzasi, D. L., Catanzarite, J., Laher, R., Conrow, T., Shupe, D., Gautier III, T. N., \& Kreidl, T. 2000, ApJ, 532, L133

Christensen-Dalsgaard, J. 1984, in Workshop on Space Research in Stellar Activity and Variability, ed. A. Mangeney \& F. Praderie (Meudon: Observatoire de Paris), 11

Gough, D. O. 1985, Nature, 314, 14

Gough, D. O. 1996, The Observatory, 116, 313. Erratum: 117, 72

Guzik, J. A. 2000, in Variable Stars as Essential Astrophysical Tools, ed. C. Ibanoglu (Dordrecht: Kluwer), 213

Handler, G. 2000, in ASP Conf. Ser. Vol. 203, IAU Coll. 176: The Impact of Large-Scale Surveys on Pulsating Star Research, ed. L. Szabados \& D. Kurtz (San Francisco: ASP), 408

Heasley, J. N., Janes, K., Labonte, B., Guenther, D., Mickey, D., \& Demarque, P. 1996, PASP, 108, 385

Høg, E., \& Petersen, J. O. 1997, A\&A, 323, 827

Kjeldsen, H., \& Bedding, T. R. 1995, A\&A, 293, 87

Kjeldsen, H., Bedding, T. R., Viskum, M., \& Frandsen, S. 1995, AJ, 109, 1313

Kjeldsen, H., Bedding, T. R., \& Christensen-Dalsgaard, J., 2000, in ASP Conf. Ser. Vol. 203, IAU Coll. 176: The Impact of Large-Scale Surveys on Pulsating Star Research, ed. L. Szabados \& D. Kurtz (San Francisco: ASP), 73 (http://astro.ifa.au.dk/MONS)

Kurtz, D. W. 1986, in IAU Symp. 118, Instrumentation and Research Programmes for Small Telescopes, ed. J. B. Hearnshaw \& P. L. Cottrell (Dordrecht: Reidel), 251

Martic, M., Schmitt, J., Lebrun, J.-C., Barban, C., Connes, P., Bouchy, F., Michel, E., Baglin, A., Appourchaux, T., \& Bertaux, J.-L., 1999, A\&A, 351, 993

Matthews, J. M., Kurtz, D. W., \& Martinez, P. 1999, ApJ, 511, 422

Matthews, J. M. et al. 2000, in ASP Conf. Ser. Vol. 203, IAU Coll. 176: The Impact of Large-Scale Surveys on Pulsating Star Research, ed. L. Szabados \& D. Kurtz (San Francisco: ASP), 74 (http://www.astro.ubc.ca/MOST)

McNamara, D. 1997, PASP, 109, 1221

McNamara, D. H. 2000, PASP, 112, 1096

Petersen, J. O. 1998, in The First MONS Workshop: Science with a Small Space Telescope, ed. H. Kjeldsen \& T. R. Bedding (Aarhus: Aarhus Universitet), 119

Petersen, J. O., \& Høg, E. 1998, A\&A, 331, 989

Trimble, V. 1995, PASP, 107, 1012 